\title{
Cubic Invariant Spherical Surface Harmonics in Conjunction with Diffraction Strain Pole-Figures
}

\author{
C. M. BRAKMAN
}

Laboratory for Metallurgy, Delft University of Technology, Rotterdamseweg 137, 2628 AL Delft, The Netherlands

(Received January 15, 1985)

Four kinds of cubic invariant spherical surface harmonics are introduced. It has been shown previously that these harmonics occur in the equations relating measured diffraction (line-shift) elastic strain and macro-stresses generating these strains for the case of textured cubic materials. As a consequence, these harmonics are important for the determination of unknown macro-stress tensor components if the o.d.f. expansion coefficients are known. On the other hand, they play a role in the determination of unknown o.d.f. expansion coefficients if the macro-stresses are known using for instance, a tensile test device on the diffractometer. Then, even the odd-order o.d.f. expansion coefficients can be obtained. In this paper, special attention is given to the mathematical construction of the cubic harmonics, the physical symmetry requirements they are subject to and some examples are given exhibiting both even and odd order harmonics.

\section{INTRODUCTION}

It has been recently shown (van Baal, 1983; Brakman, 1983; Brakman 1984a) that the "classical" $\sin ^{2} \psi$-law (Dölle, 1979) used for residual macro-stress determination may be adapted for the case of textured materials using relevant orientation distribution function (o.d.f.) theory. It is common practice in residual stress analysis to denote the deviation angle from the specimen's surface normal by the angle $\psi$ whereas the angle $\phi$ measures the angular distance in the specimen's surface (Fig. 1). For only $\sigma_{11}$ and $\sigma_{22} \neq 0$ and texture-free cubic materials the 


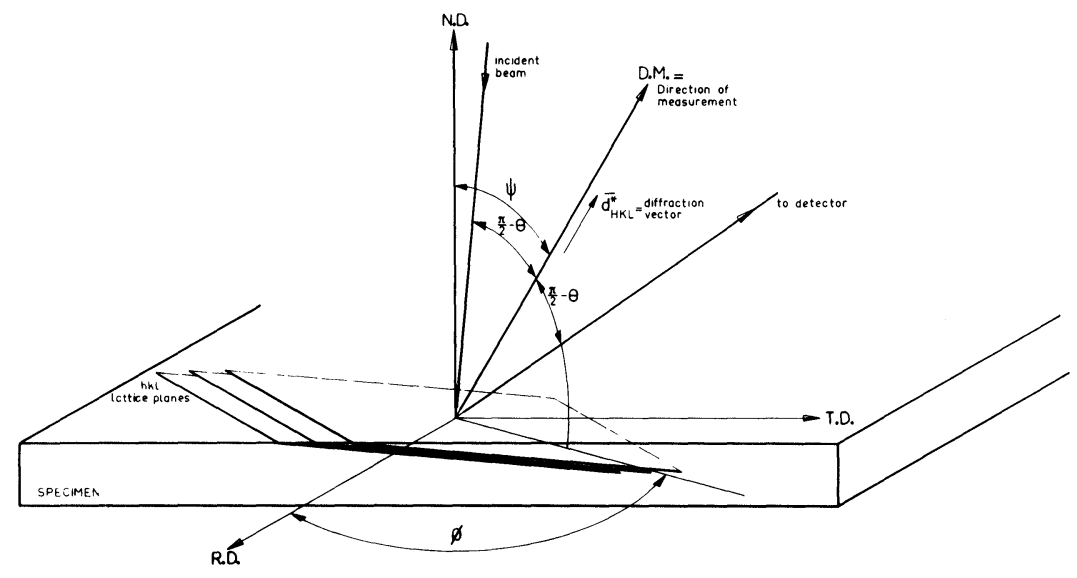

FIGURE 1 Experimental arrangement.

equation relating the measured diffraction (line-shift) elastic strain $\left\langle\varepsilon_{z z}^{\prime}\right\rangle$ and these stresses reads:

$$
\left\langle\varepsilon_{z z}^{\prime}(\psi, \phi)\right\rangle=s_{1}(h k l)\left[\sigma_{11}+\sigma_{22}\right]+\frac{1}{2} s_{2}(h k l) \sigma_{\varphi} \sin ^{2} \psi
$$

where:

$$
\begin{aligned}
s_{1}(h k l) & =s_{1122}+s_{0} \Gamma(h k l) \\
\frac{1}{2} s_{2}(h k l) & =s_{1111}-s_{1122}-3 s_{0} \Gamma(h k l) \\
\Gamma(h k l) & =h^{2} k^{2}+h^{2} l^{2}+k^{2} l^{2}
\end{aligned}
$$

(in all relevant equations it has been assumed that: $h^{2}+k^{2}+l^{2}=1$ )

$$
\begin{aligned}
& s_{0}=s_{1111}-s_{1122}-2 s_{1212} \\
& \sigma_{\phi}=\sigma_{11} \cos ^{2} \phi+\sigma_{22} \sin ^{2} \phi .
\end{aligned}
$$

Here the Reuss-model of poly-crystalline elastic crystal coupling has been used and $s_{1111}, s_{1122}$ and $s_{1212}$ are the well-known single (cubic) crystal elastic compliances. Note the predicted linear behaviour between $\left\langle\varepsilon_{z z}^{\prime}\right\rangle$ and $\sin ^{2} \psi$. For the case of a specimen exhibiting crystallographic texture and residual macro-stresses (for instance, cold-rolled steel sheet samples with orthorhombic specimen symmetry) it has been shown 
(Brakman, 1984a) that Eq. (1) may be modified using o.d.f. theory yielding:

$$
\begin{aligned}
&\left\langle\varepsilon_{z z}^{\prime}(\psi, \Phi)\right\rangle=s_{1}(h k l)\left[\sigma_{11}+\sigma_{22}\right]+\frac{1}{2} s_{2}(h k l) \sigma_{\Phi} \sin ^{2} \psi \\
&+ \frac{-s_{0}}{2 P_{h k l}(\psi, \Phi)}\left\{\left(\sigma_{11}-\sigma_{22}\right) \cos 2 \Phi-\sigma_{\Phi} \sin ^{2} \psi\right\} \\
& \times \sum_{\substack{j=4 \\
\mu=1}}\left[F_{1}(h k l, j, \mu) B_{j}^{\mu}(\psi, \Phi)+F_{2}(h k l, j, \mu) C_{j}^{\mu}(\psi, \Phi)\right] \\
&+\frac{+s_{0}}{2 P_{h k l}(\psi, \Phi)}\left(\sigma_{11}-\sigma_{22}\right) \sin 2 \Phi \cos \psi \\
& \times \sum_{\substack{j=4 \\
\mu=1}}\left[-F_{1}(h k l, j, \mu) C_{j}^{\mu}(\psi, \Phi)+F_{2}(h k l, j, \mu) B_{j}^{\mu}(\psi, \Phi)\right] \\
&+\frac{+s_{0}}{2 P_{h k l}(\psi, \Phi)} \sigma_{\Phi} \sin 2 \psi \\
& \times \sum_{\substack{j=4 \\
\mu=1}}\left[F_{3}(h k l, j, \mu) D_{j}^{\mu}(\psi, \Phi)+F_{4}(h k l, j, \mu) E_{j}^{\mu}(\psi, \Phi)\right] \\
&+ \frac{+s_{0}}{2 P_{h k l}(\psi, \Phi)}\left(\sigma_{11}-\sigma_{22}\right) \sin 2 \Phi \sin \psi \\
& \sum_{\substack{j=4 \\
\mu=1}}\left[F_{3}(h k l, j, \mu) E_{j}^{\mu}(\psi, \Phi)-F_{4}(h k l, j, \mu) D_{j}^{\mu}(\psi, \Phi)\right]
\end{aligned}
$$

where the series-expansion method of the o.d.f. on the rotation group in $R_{3}$ has been used cf. the theory given by Bunge (1982, pp. 47-118).

$$
\begin{gathered}
P_{h k l}(\psi, \phi)=\text { normalized (in terms of "times random") intensity } \\
\text { of the }\{h k l\} \text {-polefigure in the } \psi, \phi \text {-direction. }
\end{gathered}
$$

The functions $B_{j}^{\mu}(\psi, \phi), C_{j}^{\mu}(\psi, \phi), D_{j}^{\mu}(\psi, \phi)$ and $E_{j}^{\mu}(\psi, \phi)$ are defined in Eqs. (I-1) through (I-4) of the Appendix. They all contain the well-known o.d.f. expansion coefficients $C_{j}^{\mu \nu}$. Note that the strain $\left\langle\varepsilon_{z z}^{\prime}\right\rangle$ is not the strain in the $\psi, \phi$-direction of the specimen as a whole but rather the strain of the set of crystals having their orientation such that one of their $\{h k l\}$ lattice-plane normals, say $[h k l]$ coincides with the $\psi, \phi$ direction. Hence, for the general case the measured strain depends on the $\{h k l\}$ reflection used for a given stress-state. The functions $F_{1}$ 
through $F_{4}$ in Eq. (2) are the spherical surface harmonics given special attention here. Their definition reads:

$$
\begin{aligned}
F_{1}(h k l, j, \mu)= & \left(a_{2}^{2}+b_{2}^{2}\right) \\
& \times \sum_{m=-j}^{j} \dot{A}_{j}^{m \mu} P_{j}^{m 2}\left(\cos \phi_{B}\right) \cos \left[m \beta_{B}+2 \arg Z_{2}(h k l)\right] \\
F_{2}(h k l, j, \mu)= & \left(a_{2}^{2}+b_{2}^{2}\right)^{\frac{1}{2}} \\
& \times \sum_{m=-j}^{j} \dot{A}_{j}^{m \mu} P_{j}^{m 2}\left(\cos \phi_{B}\right) \sin \left[m \beta_{B}+2 \arg Z_{2}(h k l)\right] \\
-F_{3}(h k l, j, \mu)= & \left(a_{1}^{2}+b_{1}^{2}\right)^{\frac{1}{2}} \\
& \times \sum_{m=-j}^{j} \dot{A}_{j}^{m \mu} P_{j}^{m 1}\left(\cos \phi_{B}\right) \sin \left[m \beta_{B}-\arg Z_{1}(h k l)\right] \\
F_{4}(h k l, j, \mu)= & \left(a_{1}^{2}+b_{1}^{2}\right)^{\frac{1}{2}} \\
& \quad \times \sum_{m=-j}^{j} \dot{A}_{j}^{m \mu} P_{j}^{m 1}\left(\cos \phi_{B}\right) \cos \left[m \beta_{B}-\arg Z_{1}(h k l)\right]
\end{aligned}
$$

An example of each of these functions is given in Figs. 2 through 5 where both even and odd $j$ have been used.

The coefficients $\dot{A}_{j}^{m \mu}$ are the well-known cubic symmetry coefficients as introduced by Bunge (1982, pp. 384 and 501) and the functions $P_{j}^{m 1}$ and $P_{j}^{m 2}$ are defined by Bunge $(1982$, p. 351$)$

$$
\begin{aligned}
& \phi_{B}=\arccos (l) \\
& \beta_{B}=\arccos \left(h\left(h^{2}+k^{2}\right)^{-\frac{1}{2}}\right)
\end{aligned}
$$

These angles are used as given by Bunge (1982, p. 371)

$$
\begin{aligned}
& a_{1}=h k\left(h^{2}-k^{2}\right)\left(h^{2}+k^{2}\right)^{-\frac{1}{2}} \\
& b_{1}=l\left(-h^{4}-k^{4}+h^{2} l^{2}+k^{2} l^{2}\right)\left(h^{2}+k^{2}\right)^{-\frac{1}{2}} \\
& a_{2}=\left(h^{4}\left(k^{2}-l^{2}\right)+k^{4}\left(h^{2}-l^{2}\right)\right)\left(h^{2}+k^{2}\right)^{-\frac{1}{2}} \\
& b_{2}=h k l\left(h^{2}-k^{2}\right)\left(h^{2}+k^{2}\right)^{-\frac{1}{2}}
\end{aligned}
$$

$\arg Z_{1}(h k l)=\operatorname{arctg} b_{1} / a_{1}=\operatorname{arct} \operatorname{tg}\left(l\left(-h^{4}-k^{4}\left(+h^{2} l^{2}+k^{2} /^{2}\right) h k\right.\right.$ $\left.\left(h^{2}-k^{2}\right)\right)$

$\arg Z_{2}(h k l)=\frac{1}{2} \operatorname{arctg} b_{2} / a_{2}$

$$
=\frac{1}{2} \operatorname{arctg}\left(h k l\left(h^{2}-k^{2}\right) /\left(h^{4}\left(k^{2}-l^{2}\right)+k^{4}\left(h^{2}-l^{2}\right)\right)\right)
$$




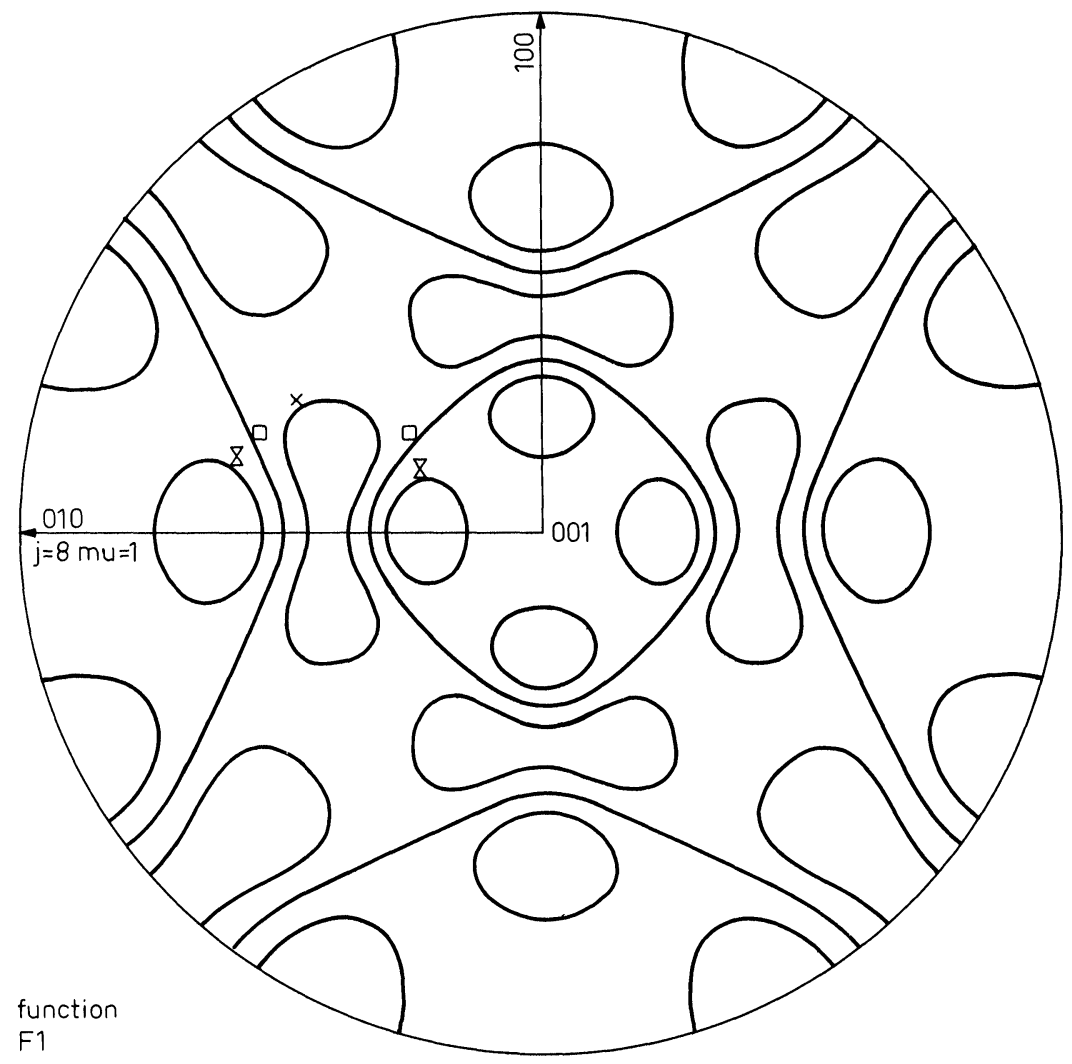

FIGURE 2 Function $F_{1}$ for $j=8$ and $\mu=1$.

It may be shown (Penning, 1984) that:

$$
\begin{aligned}
& a_{1}^{2}+b_{1}^{2}=3 h^{2} k^{2} l^{2}+\Gamma(h k l)-4 \Gamma^{2}(h k l) \\
& a_{2}^{2}+b_{2}^{2}=-3 h^{2} k^{2} l^{2}+\Gamma^{2}(h k l)
\end{aligned}
$$

$\arg Z_{1}(h k l)+\arg Z_{2}(h k l)=h k l$-permutation independent

Note that: $F_{1} \equiv 0$ for the $\{h o o\}$ and $\{h h h\}$ reflections and $F_{2}=F_{3}=$ $F_{4} \equiv 0$ for the $\{h o o\},\{h h o\}$ and $\{h h h\}$ reflections.

Note that it follows using Eq. (2) that for the $\{h o o\}$ and $\{h h h\}$ reflec- 


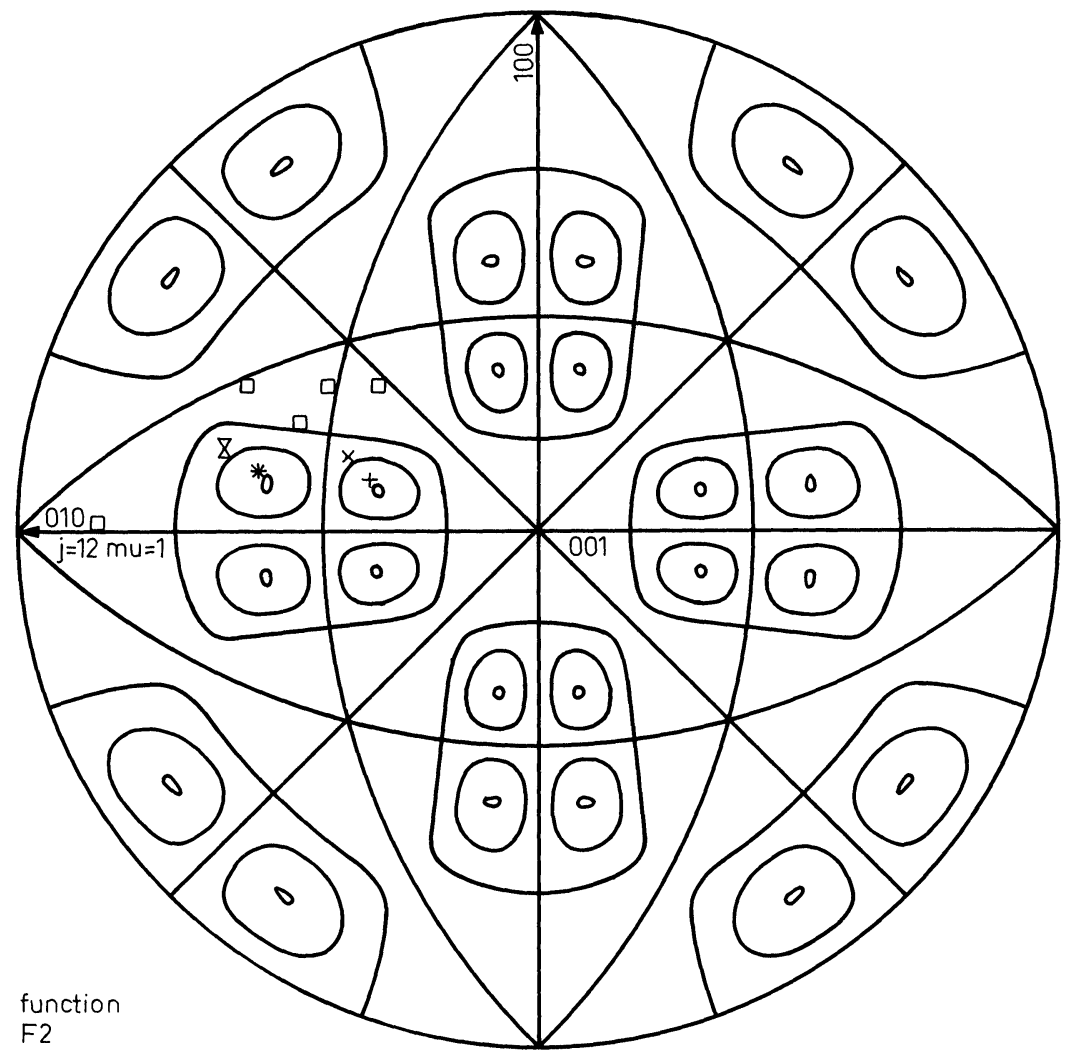

FIGURE 3 Function $F_{2}$ for $j=12$ and $\mu=1$.

tions the relevant expressions for $\left\langle\varepsilon_{z z}^{\prime}\right\rangle$ do not exhibit any texturedependency at all if the Reuss-model is taken to be valid.

Note that Eq. (2) exhibits in principle, both even and odd $j$.

Note also that function $F_{1}$ and $F_{3}$ are invariant w.r.t. any permutation of $h, k$ and $l$ whereas $F_{2}$ and $F_{4}$ develop a negative sign for 24 of the 48 possible permutations of $h, k$ and $l$. See for the latter case Figs. 3 and 5 whereas the former is demonstrated in Figs. 2 and 4. 


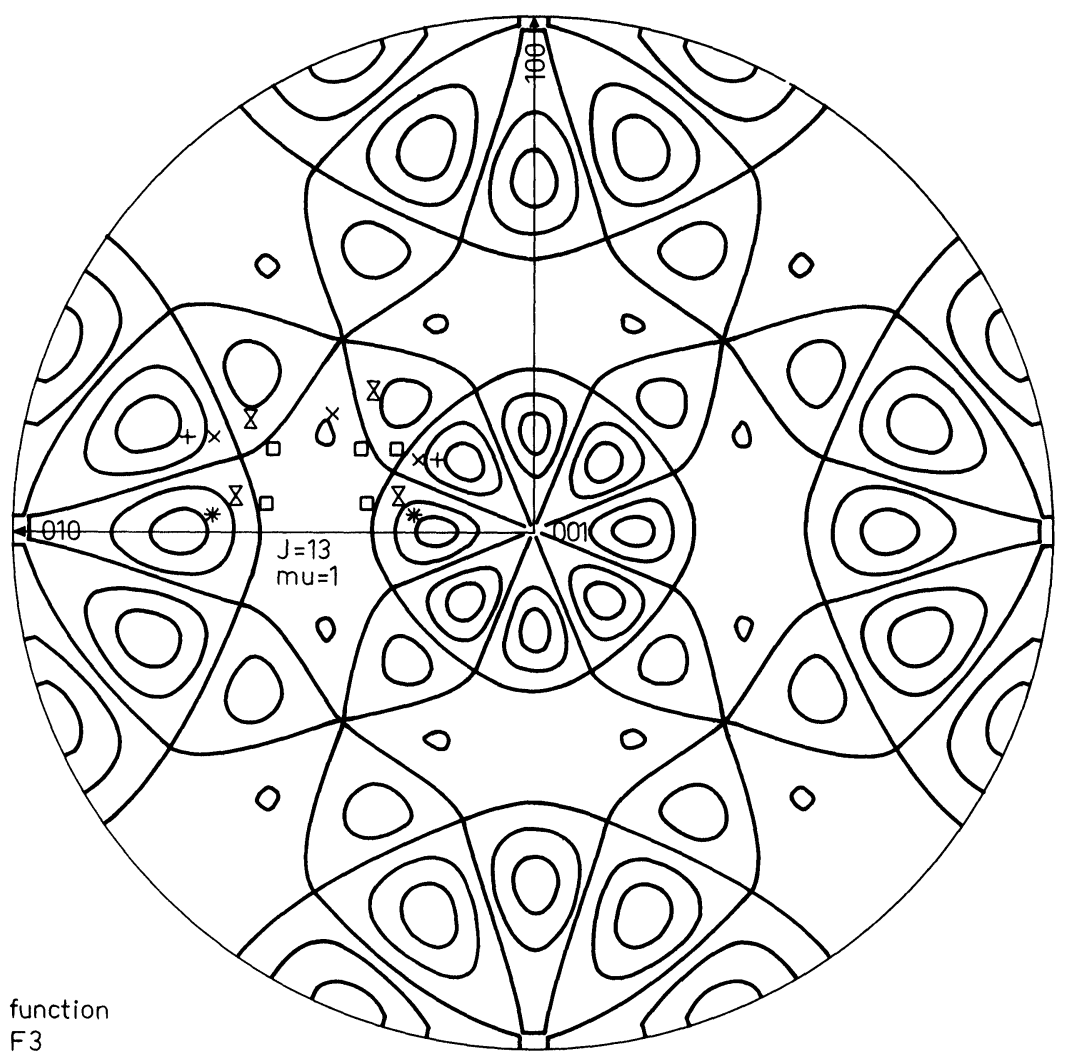

FIGURE 4 Function $F_{3}$ for $j=13$ and $\mu=1$.

\section{PHYSICAL SYMMETRY CONSIDERATIONS}

\section{Centro-symmetry of the diffraction strain pole-figure} $\left\langle\varepsilon_{z z}^{\prime}(\psi, \phi)\right\rangle$

Since the diffraction strain $\left\langle\varepsilon_{z z}^{\prime}\right\rangle$ is a physical quantity depending on the angles $\psi$ and $\phi$ it can be represented for a given (macro) stress- 


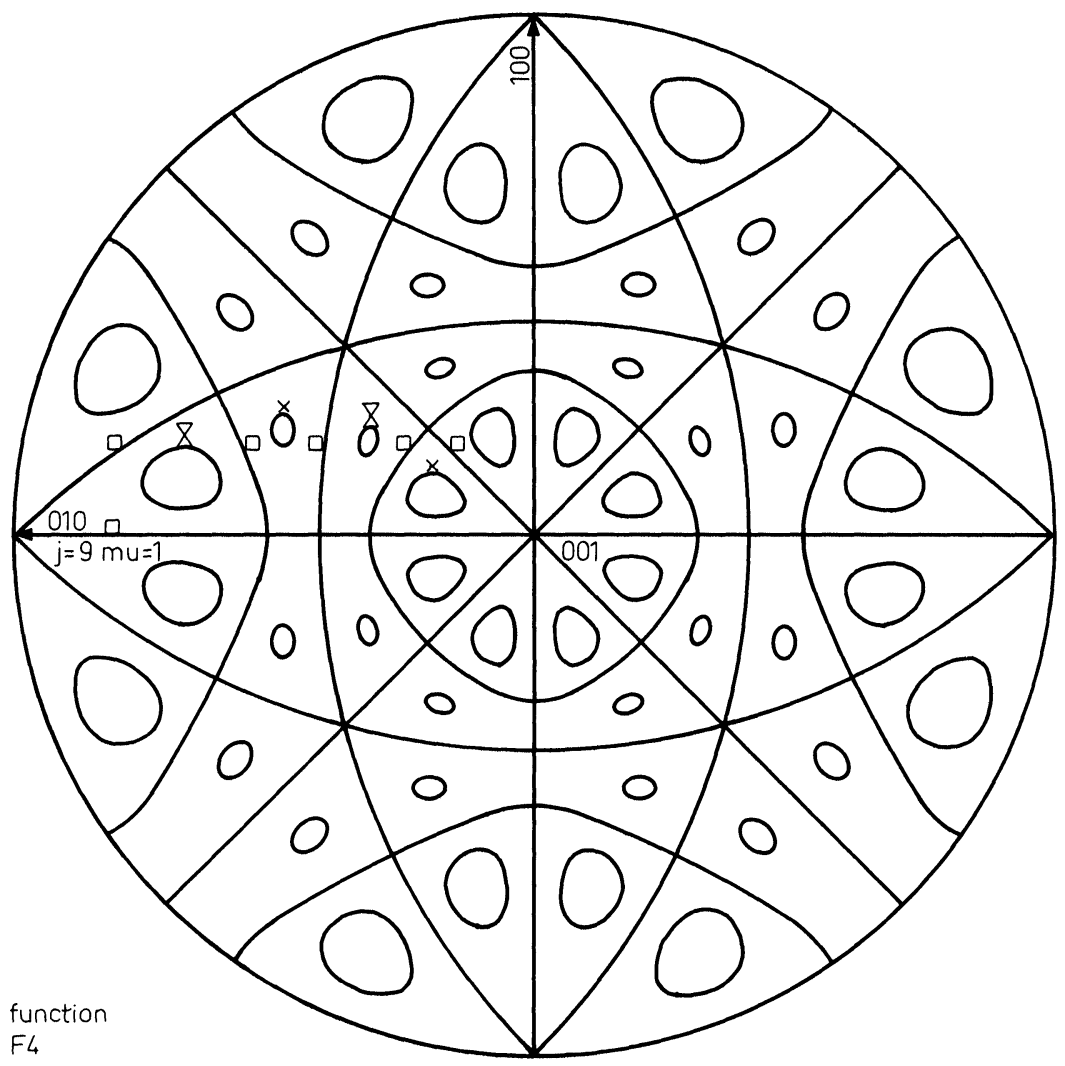

FIGURE 5 Function $F_{4}$ for $j=9$ and $\mu=1$.

The iso-density lines in Figures 2 thr. 5 are: +-0.07

$$
\begin{array}{rr}
\times & -0.03 \\
\square & 0.00 \\
\nabla & 0.03 \\
* & 0.07
\end{array}
$$

state in terms of a diffraction strain pole-figure as introduced by Hoffman et al. (1983) and also by Brakman (1984a). From Friedel's law it follows that, similar to "conventional" diffraction intensity polefigures, these pole-figures should exhibit centro-symmetry, that is the following should hold:

$$
\left\langle\varepsilon_{z z}^{\prime}(\pi-\psi, \pi+\phi)\right\rangle=\left\langle\varepsilon_{z z}^{\prime}(\psi, \phi)\right\rangle
$$


Using Eqs. (I-1) through (I-4) (see Appendix), it follows from Eq. (2) that this is not the case.

Analysis reveals that just like the case for the "conventional" diffraction intensity pole-figures (Bunge, 1982, p. 106) the contributions due to crystals exhibiting a [hkl] vector in the $\psi, \phi$ direction should be taken into account in addition to [hkl]. This problem has been discussed extensively elsewhere (Brakman, 1984b) where it was found that treating the 2 orientations exhibiting a $[\mathrm{hkl}]$ and a [hkl] vector in the $\psi, \phi$ direction respectively, is sufficient. Note that a [hkl] does not imply an inversion of cubic crystal reference axes: the choice of [hkl] as a vector parallel to the $\psi, \phi$ direction generates a physically different crystallite orientation within the context of this problem (Brakman, 1984a).

Then it follows, assuming equal structure factors of these two orientations, that the functions $F_{2}$ and $F_{4}$ vanish from Eq. (2).

The correct expression is immediately obtained by then omitting all terms exhibiting $F_{2}$ and $F_{4}$ in Eq. (2).

It may be inferred (see the Appendix) that centro-symmetry of $\left\langle\varepsilon_{z z}^{\prime}\right\rangle$ (Eq. (5)) is fulfilled then. Since $\left\langle\varepsilon_{z z}^{\prime}\right\rangle$ is a physical quantity it is also required that the eventual equation be invariant w.r.t. any permutation of $h$, $k$ and $l$. Since only the functions $F_{1}$ and $F_{3}$ remain this is indeed the case. Note that functions $F_{2}$ and $F_{4}$ vanish from Eq. (2) for both even and odd $j$ whereas functions $F_{1}$ and $F_{3}$ persist for both even and odd $j$ values. Hence, it can be argued that the "selection-rule" obtained from the requirement cf. Eq. (5) is the vanishing of $F_{2}$ and $F_{4}$ from the eventual equation rather than imposing a restriction upon the permissible values of $j$ as is the case with the diffraction intensity pole-figures (Bunge, 1982, p. 106).

Note that for the $\{h h k\}$ and $\{h k o\}$ reflections the functions $F_{2}$ and $F_{4}$ may be shown to be equal to zero (as was also the case for the $\{h o o\}$, $\{h h o\}$ and $\{h h h\}$ reflections). This may also be seen from Figs. 3 and 5. In addition to this it can be shown (Brakman, 1984b) that for the complete treatment of Eq. (2) for these reflections the consideration of the crystallite orientations associated with [hhk], or [hko] is superfluous due to the "symmetrical" nature of these directions. It can be shown that they yield exactly the same result as follows from [hhk] and [hko] respectively. $\dagger$ Consequently, for the determination of $\left\langle\varepsilon_{z z}^{\prime}\right\rangle$ (if these

$\dagger$ In fact, it can be shown that the treatment of both orientations following from the choice of $[h k l]$ and $[h k l]$ as vectors parallel to the $\psi, \phi$ direction is only necessary for those reflections where $h, k$, and $l$ are all different from each other i.e. for instance, the b.c.c. $\{321\},\{651\}$ reflections etc. 
reflections are used) the treatment of only $[h k k]$ or [hko] respectively is sufficient omitting the terms exhibiting $F_{2}$ and $F_{4}$ in Eq. (2).

\section{Orthorhombic symmetry of the diffraction strain pole-figure cf. Eq. (2)}

Using Eqs. (I-1) through (I-4) (see Appendix) it follows that Eq. (2) (minus the $F_{2^{-}}$and $F_{4}$-contributions) exhibits orthorhombic symmetry cf. point-group $\mathrm{mmm}$ i.e.:

$$
\begin{aligned}
\left\langle\varepsilon_{z z}^{\prime}(\psi, \pi-\phi)\right\rangle & =\left\langle\varepsilon_{z z}^{\prime}(\psi, \pi+\phi)\right\rangle=\left\langle\varepsilon_{z z}^{\prime}(\psi, 2 \pi-\phi)\right\rangle \\
& =\left\langle\varepsilon_{z z}^{\prime}(\pi-\psi, \phi)\right\rangle=\left\langle\varepsilon_{z z}^{\prime}(\psi, \phi)\right\rangle
\end{aligned}
$$

Note that this can only be true for a specimen exhibiting a diffaction intensity pole-figure cf. to point group $\mathrm{mmm}$ where it should hold:

$$
\begin{aligned}
P_{h k l}(\psi, \pi-\phi) & =P_{h k l}(\psi, \pi+\phi)=P_{h k l}(\psi, 2 \pi-\phi) \\
& =P_{h k l}(\pi-\psi, \phi)=P_{h k l}(\psi, \phi)
\end{aligned}
$$

Note that for the diffraction intensity pole-figure centro-symmetry is fulfilled as well:

$$
P_{h k l}(\pi-\psi, \pi+\phi)=P_{h k l}(\psi, \phi)
$$

\section{Cubic crystal symmetry}

The cubic symmetry coefficients $\dot{A}_{j}^{m \mu}$ cf. Eqs. (3a) through (3d) are defined as a consequence of point-group 432:

A 4-fold rotation axis parallel to [001] yields that $m$ in Eqs. (3a) through (3d) should be equal to a multiple of 4, the 3-fold rotation axis parallel to [111] leads to the well-known relationship that the coefficients $\dot{A}_{j}^{m \mu}$ must satisfy i.e.

$$
\dot{A}_{j}^{s \mu}=\sum_{m=-j}^{j} \dot{A}_{j}^{m \mu} P_{j}^{m s}\left(\cos \frac{\pi}{2}\right)
$$

for both even and odd $j$ and a 2 -fold rotation axis parallel to [100] yields:

$$
\dot{A}_{j}^{-m \mu}=(-1)^{j} \dot{A}_{j}^{m \mu}
$$

The other symmetry elements of 432 do not yield additional useful relationships w.r.t. the context of this paper. 
As such, rotations only are needed which comply with the seriesdevelopment method of the o.d.f. on the rotation group in $R_{3}$ as has been introduced by Bunge (1982, pp. 47-118).

However, upon considering point-group $m 3 m$ a centre of inversion occurs which cannot be accounted for using rotations only.

It has however, been shown (Brakman, 1984b) that due to this centre of inversion all physically relevant crystallite-orientations can be "reached" using rotations only. Consequently, Eq. (2) (minus the $F_{2}$ and $F_{4}$-contributions) should represent physical reality (the Reussmodel taken to be valid) for textured materials consisting of cubic crystals exhibiting symmetries cf. point-groups 432 or $m 3 m$.

One can distinguish now between 3 cases:

(i) crystal symmetry cf. point-group $m 3 m$ and specimen symmetry cf. $\mathrm{mmm}$. In this case the right-handed and left-handed o.d.f.'s are both equal and (equivalent) to the o.d.f. (Bunge, 1982, p. 105). The treatment given here concerns this o.d.f. and use of rotations only (as has been used in the derivation of Eq. (2)) is sufficient.

Note that specimen symmetry cf. point-group 222 can not occur if the crystals satisfy $m 3 m$.

(ii) crystal symmetry cf. point-group 432 and specimen symmetry cf. $\mathrm{mmm}$. In this case the right- and left-handed o.d.f.'s are equal to each other (the o.d.f. does not exist here) and the o.d.f.-coefficients $C_{j}^{\mu \nu}$ as they occur in Eq. (2) can be regarded as:

$$
C_{j}^{\mu \nu}=\frac{1}{2}\left[C_{j}^{L \mu \nu}+C_{j}^{R \mu \nu}\right] \text { where } C_{j}^{L \mu \nu}=C_{j}^{R \mu \nu}
$$

cf. Eq. (4.303) from Bunge (1982). Equation (2) is still adequate then.

(iii) crystal symmetry cf. point-group 432 and specimen symmetry cf. 222. In this case the right- and left-handed o.d.f.'s are different from each other (possibly in every orientation $g$ ) and they have to be treated separately. However, right- and left-handedness has to be defined with respect to some physical property. For polycrystalline diffraction experiments and measurement of elements of 2 nd order (strain) tensors (as is the case here) it can be expected that measurements obtain contributions from both right- and left-handed o.d.f.'s at the same time. This case is treated in Eq. (4.323) from Bunge (1982) for polycrystalline diffraction experiments. For the case of diffraction strain pole figures the o.d.f. coefficients $C_{j}^{\mu v}$ as they occur in Eq. (2) have to be substituted by: $M^{R} C_{j}^{R^{\mu \nu}}+M^{L} C_{j}^{L^{\mu \nu}}$ where $M^{R}$ and $M^{L}$ denote the fractions of the right- and left-handed crystals 
respectively. The odd order $C_{j}^{\mu \nu}$ do not drop from Eq. (2). Consequently, the virtual o.d.f. giving rise to the measured diffraction strain pole-figure is different from the one yielding the measured intensity pole-figure. Eq. (2) is still adequate.

\section{DISCUSSION}

It can be seen from Figures 3 and 5 that functions $F_{2}$ and $F_{4}$ exhibit a symmetry cf. point-group 432 for both even and odd $j$.

In this way they resemble the "normal" cubic invariant spherical surface harmonics as defined by Bunge (1982, pp. 384 and 567):

$$
\dot{K}_{j}^{\mu}\left(\phi_{B}, \beta_{B}\right)=(2 \pi)^{-\frac{1}{2}} \sum_{m=-j}^{j} \dot{A}_{j}^{m \mu} \overline{P_{j}^{m}\left(\cos \phi_{B}\right)} \exp \left(\operatorname{im} \beta_{B}\right)
$$

for $o d d j$. These functions cannot satisfy $m 3 m$ for odd $j$ as analysis shows. On the other hand, functions $F_{1}$ and $F_{3}$ (Figures 2 and 4 depict examples of them for both even and odd $j$ ) satisfy $m 3 m$ for both even and odd $j$.

As such their behaviour resembles the "normal" harmonics cf. Eq. (10) for even $j$.

It has been shown by Bunge (1982, pp. 100-107) that for the case of diffraction intensity pole figures the treatment of the crystalliteorientations following from taking [hkl] and [hkl] parallel to the $\psi, \phi$ direction leads to the occurrence in the eventual equations for the pole figure intensity of a factor equal to:

$$
\left[1+(-1)^{j}\right]
$$

As a consequence, the o.d.f. expansion coefficients $C_{j}^{\mu v}$ cannot be determined for odd $j$ from those pole-figures.

It is argued now that for the case of diffraction strain pole figures a different situation develops. It has been obtained (Brakman, 1984b) that instead of Eq. (11) the following factors then occur in the eventual equations:

$$
\begin{aligned}
& {\left[1+(-1)^{2 j}\right] \text { for } F_{1} \text { and } F_{3}} \\
& {\left[1+(-1)^{2 j+1}\right] \text { for } F_{2} \text { and } F_{4}}
\end{aligned}
$$

which complies with the vanishing of $F_{2}$ and $F_{4}$ as mentioned previously. Hence, it follows using Eqs. (I-1) thr. (I-4) that the o.d.f. 
expansion coefficients $C_{j}^{\mu v}$ are not undeterminable for odd $j$ from diffraction (line-shift) elastic strain pole-figures.

Note that the "selection rule": $C_{j}^{\mu \nu}$ undeterminable for odd $j$ for the case of diffraction intensity pole-figures is replaced here by the vanishing of functions $F_{2}$ and $F_{4}$ for both even and odd $j$.

Note that for all hkl: $F_{2}=F_{4} \equiv 0$ for $j=4$ and $F_{2}=0$ for $j=8$.

\section{CONCLUSIONS}

(i) Four kinds of cubic invariant spherical surface harmonics have been introduced. Two of them (functions $F_{1}$ and $F_{3}$ ) satisfy for even and odd order the symmetry-requirements of point-group $m 3 m$ whereas the other two (functions $F_{2}$ and $F_{4}$ ) comply with point-group 432 for even and odd $j$. For the treatment of diffraction strain polefigures the functions $F_{2}$ and $F_{4}$ are not necessary (except for the case of anomalous scattering).

(ii) the functions derived may serve as a tool for the determination of unknown macro-stresses in textured cubic materials exhibiting residual or applied stress if the o.d.f. expansion coefficients are known. On the other hand, if the macro-stresses are known these functions may be used to obtain the o.d.f. expansion coefficients $C_{j}^{\mu \nu}$ for both even and odd $j$ provided enough diffraction strain measurements are available.

\section{References}

Baal, C. M. van. Phys. Stat. Sol. (a)77, 521-526 (1983).

Brakman, C. M. J. Appl. Cryst. 16, 325-340 (1983).

Brakman, C. M. Cryst. Res. Technol, in press (1984a).

Brakman, C. M. J. Appl. Cryst., submitted (1984b).

Bunge, H. J. Texture Analysis in Materials Science, London, Butterworths (1982).

Dölle, H. J. Appl. Cryst. 12, 489-501 (1979).

Hoffmann, J., Neff, H., Scholtes, B. and Macherauch, E. Härterei-Tech. Mitt. 38, 180-183 (1983).

Penning, P. private communication (1984). 


\section{APPENDIX}

The functions $C_{j}^{\mu}(\psi, \phi)$ thr. $E_{j}^{\mu}(\psi, \phi)$ are defined as follows:

$$
\begin{aligned}
B_{j}^{\mu}(\psi, \phi) & =\sum_{v=1}^{N(i)} C_{j}^{\mu v}(-1)^{v-1} \sqrt{2-\delta_{v, 1}} \\
\times & {\left[P_{j}^{2,2(v-1)}(\cos \psi)+(-1)^{j} P_{j}^{-2,2(v-1)}(\cos \psi)\right] \cos 2(v-1) \phi } \\
C_{j}^{\mu}(\psi, \phi) & =\sum_{v=1}^{N(j)} C_{j}^{\mu v}(-1)^{v-1} \sqrt{2-\delta_{v, 1}} \\
\times & {\left[P_{j}^{2,2(v-1)}(\cos \psi)-(-1)^{j} P_{j}^{-2,2(v-1)}(\cos \psi)\right] \sin 2(v-1) \phi }
\end{aligned}
$$

$D_{j}^{\mu}(\psi, \phi)=\sum_{\nu=1}^{N(j)} C_{j}^{\mu v}(-1)^{\nu-1} \sqrt{2-\delta_{v, 1}}$

$$
\times\left[P_{j}^{1,2(v-1)}(\cos \psi)+(-1)^{j} P_{j}^{-1,2(v-1)}(\cos \psi)\right] \cos 2(v-1) \phi
$$

$E_{j}^{\mu}(\psi, \phi)=\sum_{\nu=1}^{N(j)} C_{j}^{\mu \nu}(-1)^{\nu-1} \sqrt{2-\delta_{v, 1}}$

$$
\times\left[P_{j}^{1,2(v-1)}(\cos \psi)-(-1)^{j} P_{j}^{-1,2(v-1)}(\cos \psi)\right] \sin 2(v-1) \phi
$$

where $N(j)=(j+2) / 2$ ("integer division").

Note that these equations have been written for orthorhombic specimen symmetry.

Note that for instance,

$$
\begin{aligned}
& B_{j}^{\mu}(\pi-\psi, \pi+\phi)=+B_{j}^{\mu}(\psi, \phi) \\
& C_{j}^{\mu}(\pi-\psi, \pi+\phi)=-C_{j}^{\mu}(\psi, \phi) \\
& D_{j}^{\mu}(\pi-\psi, \pi+\phi)=-D_{j}^{\mu}(\psi, \phi) \\
& E_{j}^{\mu}(\pi-\psi, \pi+\phi)=+E_{j}^{\mu}(\psi, \phi)
\end{aligned}
$$

